\title{
Recent Heavy Flavor Physics Results from Fixed Target Experiments
}

\author{
L. Spiegel \\ Fermi National Accelerator Laboratory \\ P.O. Box 500, Batavia, Illinois 60510
}

November 1991

* Presented at the 3rd Topical Seminar on Heavy Flavours, San Miniato, Tuscany (Italy), June 17-21, 1991. 


\section{Disclaimer}

This report was prepared as an account of work sponsored by an agency of the United States Government. Neither the United States Government nor any agency thereof, nor any of their employees, makes any warranty, express or implied, or assumes any legal liability or responsibility for the accuracy, completeness, or usefullness of any information, apparatus, product, or process disclosed, or represents that its use would not infringe privately owned rights. Reference herein to any specific commercial product, process, or service by trade name, trademark, manufacturer, or otherwise, does not necessarily constitute or imply its endorsement, recommendation, or favoring by the United States Government or any agency thereof. The views and opinions of authors expressed herein do not necessarily state or reflect those of the United States Government or any agency thereof. 


\title{
RECENT HEAVY FLAVOR PHYSICS RESULTS FROM FIXED TARGET EXPERIMENTS
}

\author{
Leonard Spiegel \\ Fermi National Accelerator Laboratory, P.O. Box 500, Batavia, II 60510
}

Recent results from fixed target experiments in the field of heavy quark flavors, as published or otherwise disseminated in the last year, are reviewed. Emphasis is placed on distilling the main conclusions from these results.

\section{INTRODUCTION}

Not surprisingly, recent results in the field of heavy quark flavors from fixed target experiments tend to emphasize the strengths of the fixed target programs relative to the present generation of collider machines. These strengths include the ability to probe nuclear targets, the ability to measure primary and secondary vertices in a straightforward fashion, and the ability to detect and measure states for which the production cross-section is either intrinsically very small or unfavorable due to quantum number restrictions as in $\mathrm{e}^{+} \mathrm{e}^{-}$colliders. Also, the relatively low center of mass energies in hadroproduction experiments are of some benefit in the isolation of both inclusive heavy flavor signais and associatively produced heavy flavor signals. Some fixed target experiments of recent vintage have accumulated sufficient numbers of flavorantiflavor events so as to allow meaningful comparisons with Quantum Chromodynamics (QCD) predictions.

Typically these fixed target experiments share in common large spectrometers which are capable of handling high luminosities and event rates. Many of the experiments employ extensive vertex detectors often silicon microvertex detectors (SMDs), emulsions, and charged-coupled devices (CCDs) for the purposes of strengthening signal-to-noise levels and extracting lifetime measurements for the flavor changing decays. For the most part, heavy flavor for this generation of fixed target experiments is synonymous with open charm physics. There is one experiment which is reporting a handful of partially or totally reconstructed beauty events. There is also recent data on the nuclear dependence of $\Upsilon$ production.

By necessity of the energies involved. experiments which are reporting results took their data at either the Fermi National Accelerator Laboratory (FNAL) or the European Organization for Nuclear Research (CERN). For the most part this paper is concerned with results that have either been published or otherwise publicized in the last year. In many cases the experimental data upon which the results rest dates back several years and has only been brought to light by extensive analysis. Wherever possible reference is made to journal articles.

For reterence, the primary proton energy at FNAL is $800 \mathrm{GeV}$ in the fixed target program; at CERN the primary energy is $400 \mathrm{GeV}$. For the sake of brevity, FNAL experiments are prefixed by a redundant $E$ and CERN experiments by either NA (North Area) or WA (West Area) when referred to in this paper. Finally, in keeping with convention, whenever heavy flavor states are listed in this paper the inclusion of charge conjugate states is always implicitly assumed unless otherwise stated.

\section{A-DEPENDENCE PHYSICS RESULTS}

Typically nuclear dependence experiments endeavor to determine the $\alpha$ parameter as in $\sigma_{A}=$ $A^{\alpha} \sigma_{0}$ where $\sigma_{A}$ is the cross-section per nucleus (atomic mass $\mathrm{A}$ ) and $\sigma_{0}$ is the single nucleon cross- 
section. For point-tike processes $\alpha$ should be close to 1 ; for nuclear-scale cross-sections such as the total inelastic cross-section, $\alpha$ should be closer to $2 / 3$, that is $\sigma_{\mathrm{A}}$ should scale with the projected surface area. It has been appreciated for some time now that hydrogen $\left({ }^{1} \mathrm{H}\right)$ does not conform to this parametrization (although deuterium $\left({ }^{2} \mathrm{H}\right)$ does). Beyond the bulk cross-section dependence with $A$ one would also like to understand the detailed dependence on kinematic variables such as the Feynman variable $\left(x_{F}\right)$, transverse momentum $\left(p_{T}\right)$, and center of mass energy $(\sqrt{s})$. By comparing the detailed dependencies of a variety of states on these kinematic variables, some degree of discrimination may be afforded between the various models which attempt to explain suppression effects in heavy targets. There is also cross-over interest in this field as $J / \psi$ suppression has been suggested as a signature for quark-gluon-plasma formation in relativistic heavy-ion collisions ${ }^{1}$.

In the case of open charm production, experiment E769 (Tagged Photon Laboratory) has reported in a recent conference ${ }^{2}$ a value of $0.92 \pm 0.08$ for $\alpha$ in inclusive $D^{0}$ production and $0.97 \pm 0.07$ for $D^{+}$. These numbers are based on the reconstruction of $\mathrm{K}^{+}$ $\pi^{+}$and $\mathrm{K}^{-} \pi^{+} \pi^{+}$signals from $250 \mathrm{GeV}$ pion interactions with $\mathrm{Be}, \mathrm{Al}, \mathrm{Cu}$, and $\mathrm{W}$ target foils the beam simultaneously skewered all four materials). A previous result from $W A 82^{3}$, also a hadroproduction experiment, gave $\alpha=0.89 \pm 0.05 \pm 0.05$, averaged over charged and neutral $D$ mesons. There are theoretical prejudices that $\alpha$ should be close to one in open production and this is certainly the conventional choice of experiments in analyzing their cross-section data. One would hope that information on $\alpha\left(x_{F}, p_{T}\right)$ will be shortly forthcoming from the recent open charm experiments.

Closed charm nuclear-dependence experiments tend to agree upon a clear deviation from unity for the $\alpha$ parameter and with their statistical advantages are able to offer more detailed information on $x_{F}$ and $P_{T}$ dependencies. Recently, for example, E672 has reported 4 a value of $\alpha=0.85 \pm 0.06$ for $\mathrm{J} / \psi$ production $\left(0.1 \leq x_{F} \leq 0.8\right)$ based on $530 \mathrm{GeV} \pi^{-}$ collisions with $\mathrm{C}, \mathrm{Al}, \mathrm{Cu}$, and $\mathrm{Pb}$ targets. In comparing results from the lead and carbon targets they further conclude that there is no obvious $x_{F}$ or $p_{T}$ dependence to $\alpha$ for the $X_{F}$ and $P_{T}$ ranges to which they were sensitive. Although there are a variety of models to explain the $\mathrm{J} / \psi$ suppression effect, the authors suggest that a recent model by Brodsky and Mueller ${ }^{5}$, which attributes suppression to final-state interactions involving the $J /$ and comoving spectator partons, may be the most likely explanation.

Unlike E672, which ran in conjunction with an open geometry experiment, E772 represents a semiclosed geometry spectrometer. Based on $800 \mathrm{GeV}$ proton interactions in ${ }^{2} \mathrm{H}, \mathrm{C}, \mathrm{Ca}, \mathrm{Fe}$, and $\mathrm{W}$ targets they report in separate publications A-dependence results for Drell-Yan continuum production $6, \mathrm{~J} / \psi$ and $\psi^{\prime}$ production 7 , and $\Upsilon$ resonance (1S and combined 2S and 3S) production 8 . Results from these publications are neatly summarized in fig. 1 . In the figure, $F$ refers to the ratio of the yields for the solid targets to liquid deuterium. The value of $\alpha=0.92 \pm$ 0.008 for $\mathrm{J} / \mathrm{H}$ and $\psi^{\prime}$ production suggests that nuclear dependence effects are at least independent of the size of the final state. In contrast to E672 there is an apparent decrease in $\alpha$ with $\mathrm{x}_{\mathrm{F}}$ and an apparent increase with $\mathrm{p}_{\mathrm{T}}$ for $\mathrm{J} N$ production. By comparing their data with $200 \mathrm{GeV}$ data from NA3 they claim that there is very little dependence to $\alpha\left(x_{F}\right)$ with beam energy. The fact that $\alpha$ is falling with $x_{F}$ is inconsistent with small-x shadowing models ${ }^{9}$ as well as hadronic reinteraction suppression models of the type previously mentioned. There is a model 10 though, which is able to qualitatively explain the $x_{F}$ dependence through an assumption of intrinsic $c \mathcal{C}$ in the wave functions of the beam particles.

With some 25,000 is and partially resolved $2 S$ and $3 S \Upsilon$ decays, E772 reports similar values within errors for the 1S $(\alpha=0.962 \pm 0.006)$ and the 2S+3S $(\alpha=0.948 \pm 0.012)$ resonances. Again this apparent equality is suggestive of a lack of dependence on the 
final state size. Interestingly, $\alpha$ shows a sharp decrease for $x_{F}<0$. Why this is so is unciear but it does underscore the general need for future experiments to enrich and expand the measurable kinematic ranges.

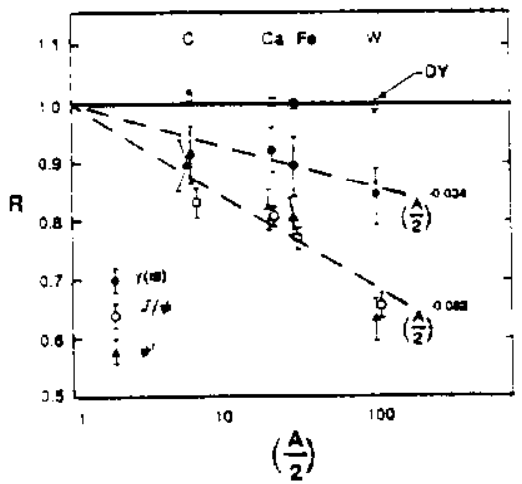

Fig. 1 - Summary of E772 $\alpha$ data

\section{PRECISION LIFETIME AND WIDTH RESULTS}

Photoproduction experiments E687 (Wide Band, $221 \mathrm{GeV}$ average photon energy) and NA14/2 (100 GeV average photon energy) have reported $11,12,13$ measurements for the $D^{0}, D^{+}, D_{s}^{+}$, and $\Lambda_{c}^{+}$mean lifetimes. In both experiments lifetime measurements are derived from $\mathrm{K}^{-} \pi^{+}$and $\mathrm{K}^{-} \pi^{+} \pi^{+} \pi^{-}$signals for the $D^{0} ; K^{-} \pi^{+} \pi^{+}$for the $D^{+} ; \phi_{s} \pi^{+}$for the $D_{s}^{+} ;$and $p K^{*} \pi^{+}$for the $\Lambda_{c}^{+}$. The impressive sizes and signal-to. background ratios of these data samples facilitated several internal consistency checks. For example, $D^{0_{1}} \mathrm{~s}$ from $D^{*}$ cascades were compared with the notag $D^{0}$ sample in the E687 analysis (see fig. 2).

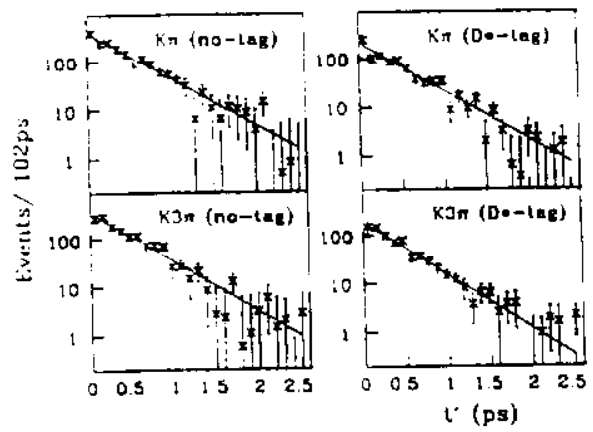

Fig. 2 - From E687 charm data
Similarly, hadroproduction experiment NA32 (ACCMOR) has reported results ${ }^{14}$ for lifetimes of the $D^{+}, D^{0}$, and $D_{s}^{+}$. These data are derived from a phase of the experiment in which $230 \mathrm{GeV} \pi^{-1} \mathrm{~s}$ were directed onto a $\mathrm{Cu}$ target. Lifetime results are based on the decay modes $K^{-} \pi^{+}$and $K^{-} \pi^{+} \pi^{+} \pi^{-}$for the $D^{0} ; K^{-}$ $\pi^{+} \pi^{+}$and $K^{-} K^{+} \pi^{+}$for the $D^{+}$; and $K^{-} K^{+} \pi^{+}$for the $D_{s}^{+}$. $A$ comparison of the lifetime results (in picoseconds) from all three experiments is shown in Table 1. These results are all in agreement with the world averages 15 , which at present are dominated by photoproduction experiment E691 (Tagged Photon Laboratory).

\begin{tabular}{|c|c|c|c|}
\cline { 2 - 4 } \multicolumn{1}{c|}{} & $E 687$ & NA14/2 & NA32 \\
\hline$\tau\left(\mathrm{D}^{+}\right)$ & $1.075 \pm 0.040 \pm 0.018$ & $1.03 \pm 0.08 \pm 0.06$ & $1.05_{-0.072}^{+0.077}$ \\
\hline$\tau\left(\mathrm{D}^{0}\right)$ & $0.424 \pm 0.011 \pm 0.007$ & $0.417 \pm .018 \pm .015$ & $0.388_{-0.021}^{+0.023}$ \\
\hline$\tau\left(\mathrm{D}_{s}^{+}\right)$ & $0.50 \pm 0.06 \pm 0.03$ & $0.33_{-0.08^{+0.03}}^{+0.12^{2}}$ & $0.469_{-0.086}^{+0.102}$ \\
\hline$\tau\left(\Lambda_{C}^{+}\right)$ & $0.20 \pm 0.03 \pm 0.03$ & $0.18 \pm 0.03 \pm 0.03$ & \\
\hline
\end{tabular}

Table 1

In contrast to all other fixed target experiments considered in this review, E760 represents a formation as opposed to hadroproduction or photoproduction experiment. Charmonium states in this experiment result from the simultaneous annihilation of all valance antiquarks of an antiproton with all valance quarks of a proton (supplied by a hydrogen gas-jet). E760 is situated in the FNAL antiproton accumulator ring. Charmonium resonances are formed by decelerating $8.9 \mathrm{GeV} / \mathrm{c}$ antiprotons to the appropriate energies. By measuring very precisely the frequency of revolution and knowing the path length of the ring, resonances are typically scanned through in $1 / 4 \mathrm{MeV}$ steps in the center of mass system. A typical scan of the $\chi_{2}$ is shown in fig. 3. Knowledge of the $J / \psi$ centroid calibrates the energy scale and this calibration is cross-checked via the $\psi^{\prime}$. Table 2 outlines 
pretiminary results for masses and widths from the scans ${ }^{16}$. The principal source of uncertainty in these results lies in the unfolding of the intrinsic momentum spread of the beam, which is comparable to the step size.

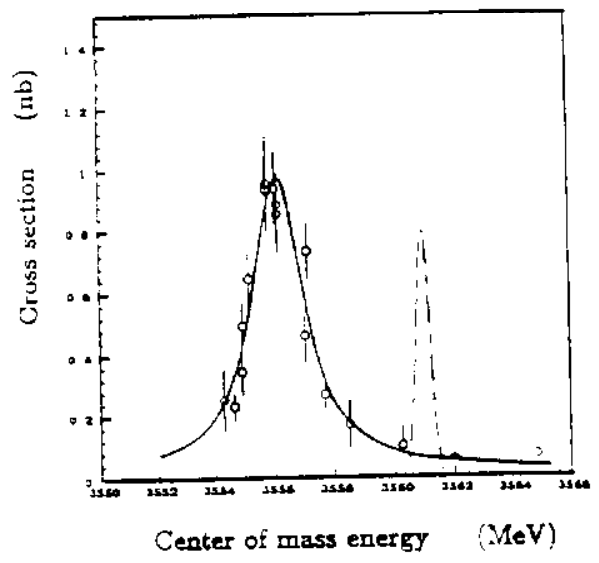

Fig. $3-E 760 \chi_{2}$ scan

\begin{tabular}{|c|c|c|}
\hline Resonance & $\begin{array}{c}\text { Mass } \\
\left(\mathrm{MeV} / \mathrm{c}^{2}\right)\end{array}$ & $\begin{array}{c}\text { Width } \\
\left(\mathrm{MeV} / \mathrm{c}^{2}\right)\end{array}$ \\
\hline$J / \psi$ & $3096.88 \pm .06 \pm .03$ & \\
\hline$\chi_{1}$ & $3510.53 \pm .04 \pm .12$ & $0.88 \pm .11 \pm .08$ \\
\hline$\chi_{2}$ & $3556.15 \pm .07 \pm .12$ & $1.98 \pm .17 \pm .07$ \\
\hline$\psi^{\prime}$ & 3686. & $0.310 \pm .05 \pm .025$ \\
\hline$\chi_{1}-\chi_{2}$ & $45.62 \pm .08 \pm .06$ & \\
\hline
\end{tabular}

Table 2

\section{HADROPRODUCTION CHARM SINGLES}

Inclusive charm production experiments tend to parameterize the differential cross-sections based on the distribution:

$$
d^{2} \sigma / d x_{F} d p_{T}^{2} \propto\left(1-\left|x_{F}\right|\right)^{n} e^{-b p_{T}^{2}} .
$$

Once the parameters $n$ and $b$ have been determined, equation (1) is used. assuming its validity either over both hemispheres or over the forward hemisphere only, to calculate the total cross-section for a particular reaction. Often, in going from cross-section per nucleus to the more universal cross-section per nucleon, the assumption is made that the crosssections are linear with atomic mass. As pointed out earlier, though, there is experimentat evidence for suppression effects in open charm production.

Based on $230 \mathrm{GeV} \pi^{-} \mathrm{Cu}$ and $\mathrm{K}^{*} \mathrm{Cu}$ interactions, NA32 (ACCMOR) has reported 17 on the properties of inclusive $D$ meson production. Charm decays were identified within a precision SMD system which was complemented by two CCDs. In all, 852 decay vertices were fully reconstructed and these include $543 D^{0} \rightarrow K^{-} \pi^{+}$and $249 D^{+} \rightarrow K^{-} \pi^{+} \pi^{+}$. From the subset of these $D^{0}$ and $D^{+}$decays which resulted from pion interactions, production parameters $n$ and $b$ are determined as:

$$
n=3.74 \pm 0.23 \quad b=0.83 \pm 0.03(\mathrm{GeV} / \mathrm{c})^{-2}
$$

The parameters are essentially identical for $D^{0}$ and $\mathrm{D}^{+}$. Assuming linear A-dependence the total crosssection is quoted as:

$$
\sigma\left(\pi^{-} N\right)=(9.5 \pm 0.4 \pm 1.9) \mu b / \text { nucleon } x_{F}>0
$$

For the $230 \mathrm{GeV}$ pions, $D^{0} \mathrm{~s}$ are apparently produced twice as frequently as $D^{+\cdot} s$.

Within a similar framework E653 reports ${ }^{18}$ on the properties of inclusive charm production in $800 \mathrm{GeV}$ proton-emulsion interactions. Their sample consists of 146 decay vertices of which less than five percent may be background. Differential distribution parameters are reported as:

$$
n=6.9_{-1.8}^{+1.9} \quad b=0.84_{-.08}^{+.10}(\mathrm{GeV} / \mathrm{c})^{-2}
$$

which translate into total inclusive cross-sections per nucleon of $38 \pm 3 \pm 13 \mu \mathrm{b}$ for the $D^{0}$ and $38 \pm 9 \pm 14$ $\mu b$ for the $D^{+}$. The cross-section is quoted for the entire range of $X_{F}$ and the differential distributions are 
observed to be centered at $x_{F}=0$, which is not unexpected given the symmetry of the reaction.

E653 further observes that their $x_{F}$ and $p_{T}$ distributions are consistent with next-to-leading-order QCD predictions for charm quarks, which leads them to conclude that the hadronization process has very little effect on the parton level kinematic distributions.

\section{HADROPRODUCTION OF CHARM PAIRS}

Experiment NA32 reports ${ }^{19}$ on a data sample of 642 events with two or more presumed charm vertices (of these, 58 seemingly represent double charm flavor pairs - a surprising result if accurate). Again, these results are based on $230 \mathrm{GeV} \pi^{-}$ interactions in a $\mathrm{Cu}$ target. Parent particles are identified as $D^{0}, D^{+}, \Lambda_{c}^{+}$, and $D_{s}^{+}$based on the number of decay tracks, the visible mass of the decay, and the presence of kaons and protons in the final state. After correcting for spectrometer acceptance the azimuthal angles $\phi_{T}$ between the parent charm particle vectors are tabulated based on the composition of the original charm-anticharm flavor state. Their results show a clear peaking at $\phi_{T}=180^{\circ}$ for $\bar{D} \bar{D}$ pairs; less so for $\Lambda_{c} \bar{D}$ and even less so for $\mathrm{O}_{\mathrm{s}} \overline{\mathrm{D}}$. Predictions from leading-order $Q C D^{20}$, which include corrections due to transverse momentum smearing in the fragmentation process, predict a significantly higher peaking at $180^{\circ}$ than is observed in the data (at the simplest level QCD would suggest a delta function at $\left.180^{\circ}\right)$. However, the experiment does point out that a hadron cluster model 21 in which the $D \bar{D}$ pair results from the decay of a $5 \mathrm{GeV}$ cluster does make predictions for the azimuthal distributions in rough agreement with the data.

In a second paper22 E653 makes qualitative comparisons with leading order QCD predictions 23 for the kinematic distributions derived from a sample of 35 charm pair events (with an estimated background of 1.4 events). As the experiment triggered on muons all of their events necessarily contain at least one semimuonic decay; it is also often the case that the unbiased, associative charm decay also contains a missing neutral. By using the measured momentum and particle identification of the visible tracks, an estimate is made for the total momentum of the original parent charm particle. An RMS error of $20-30 \%$, depending on event topology, is quoted for this procedure. For the purposes of QCD comparisons, particles are all lumped together without regard to differences in charm species.

Results form the leading order QCD predictions show reasonable agreement with the data for the invariant mass of the pair $\left(M_{c} \bar{c}\right)$, the rapidity gap $(\Delta y)$, the polar angle $(\cos \theta)$, and the Feynman variable $\left(x_{F}\right)$. Results may be summarized as:

$$
\begin{array}{lll}
M_{\propto \bar{c}} & e^{-\left(\mathrm{aM}_{\mathrm{C}} \bar{c}\right)} & a=0.53 \pm 0.14\left(\mathrm{GeV} / \mathrm{c}^{2}\right)^{-1} \\
\Delta y & e^{-\Delta \mathrm{y}^{2} / 2 \mathrm{~s}^{2}} & \mathrm{~s}=1.85 \pm 0.45 \\
\mathrm{x}_{\mathrm{F}} & \left(1-\left|\mathrm{x}_{\mathrm{F}}\right|\right)^{\mathrm{n}} & \mathrm{n}=5.4 \pm 1.4 \\
\text { PT } & e^{-\mathrm{b} p_{T}} & \mathrm{~b}=1.4 \pm 0.3(\mathrm{GeV} / \mathrm{c})^{-1}
\end{array}
$$

The $\cos \theta$ data favor, as would be expected, fusion over annihilation, although there is a stronger peaking at $\cos \theta=1$ than even the fusion model predicts (the dotted line in fig. 4). In analyzing either $p_{T}$ or $p_{T}{ }^{2}$, the paper observes that uncertainties due to the unknown transverse momentum distributions for the initial partons, higher order QCD corrections, and corrections stemming from fragmentation all preclude a straightforward comparison with their leading order QCD model. They do observe that their $\mathrm{p}^{2}$ distribution apparently has the same shape as does the $p_{T}{ }^{2}$ distribution derived from dilepton production experiments. Finally, the point is again made that, at least at this level of scrutiny, the hadronization process does not significantly alter the kinematics resulting from the fundamental parton interactions. 


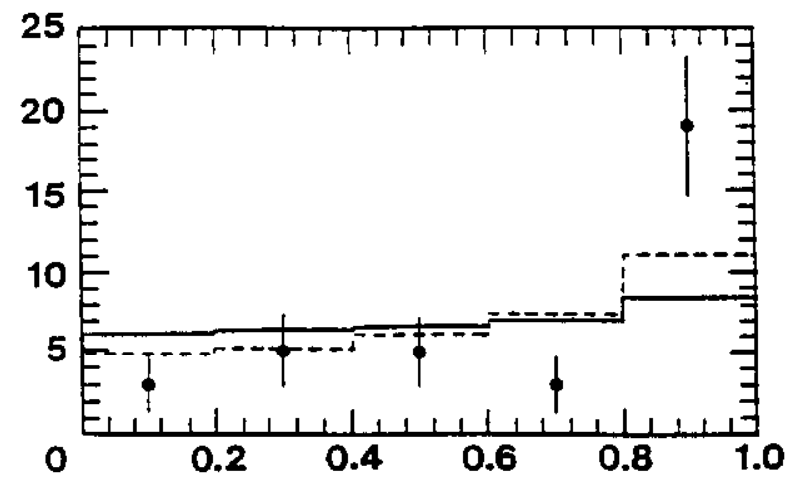

Fig. 4 - E653 charm pair $\cos \theta$ data

\section{RARE DECAY MODES}

Based on a $10^{8}$ event sample, photoproduction experiment E691 has reported in two recent publications 24,25 measurements for relative and absolute branching ratios for selected Cabibbosuppressed (strangeless) decays of $D^{0}$ and $D^{+}$ mesons. Table 3 exhibits some of the principal results. In the table CS refers to the suppressed decay and CF represents the most closely related Cabibbo-favored decay.

\begin{tabular}{|l|l|l|l|}
\cline { 2 - 4 } \multicolumn{1}{c|}{} & \multicolumn{1}{c|}{ CS } & \multicolumn{1}{c|}{ CF } & \multicolumn{1}{c|}{ CS/CF } \\
\hline$D^{0}$ & $\pi^{-} \pi^{+}$ & $K^{-} \pi^{+}$ & $.059 \pm .009 \pm .005$ \\
\hline$D^{0}$ & $K^{2} K^{+}$ & $K^{-} \pi^{+}$ & $.114 \pm .011 \pm .009$ \\
\hline$D^{0}$ & $\pi^{-} \pi^{+} \pi^{-} \pi^{+}$ & $K^{-} \pi^{+} \pi^{-} \pi^{+}$ & $.096 \pm .018 \pm .007$ \\
\hline$D^{0}$ & $K^{-} K^{+} \pi^{-} \pi^{+}$ & $K^{-} \pi^{+} \pi^{-} \pi^{+}$ & $.028_{-0.007}^{+0.008}$ \\
\hline
\end{tabular}

Table 3

Within the publications several observations are drawn concerning the results displayed in Table 3. First, it is apparent that two-body Cabibbosuppressed decay modes of the $D^{\circ}$ favor an sS pair (as opposed to no strange quarks) by about a factor of two whereas the opposite case may be true for four-body decays. The authors speculate that there may be a global balancing of the number of $s \bar{s}$ and non-ss decay modes of the $D^{0}$. Such a symmetry is consistent with spectator-diagram dominance for the suppressed decays. Secondly, there are theoretical predictions 26 of about 1.4 for the $\mathrm{K}^{-} \mathrm{K}^{+} / \pi^{-} \pi^{+}$ratio $(1.95 \pm 0.34 \pm 0.22)$ which take into account phase space differences in the decay products as well as SU(3) flavor symmetry breaking. Previous results for this ratio have all been higher although the general trend has been to approach the theoretical prediction (the ratio one computes from the most recent Particle Properties Data Booklet ${ }^{15}$ is 2.50 from the E691 result and $3 \sigma$ from the theoretical prediction).

In another publication 27 the E691 group reports on the branching ratio $D^{+} \rightarrow \bar{K}^{0} e^{+} v_{e}$, which they measure to be $.63 \pm 0.09 \pm 0.13$ relative to $D^{+} \rightarrow$ $\mathrm{K}^{-} \pi^{+} \pi^{+}$. This corresponds to an absolute branching ratio of $(5.8 \pm 0.8 \pm 1.5) \%$. Using their own measurement for the $D^{+}$lifetime they deduce the partial width $\Gamma\left(D^{+} \rightarrow \bar{K}^{0} \mathrm{e}^{+} v_{\theta}\right)$ to be $(5.3 \pm 0.7 \pm$ 1.4) $10^{10} \mathrm{~s}^{-1}$. This may be compared their previous measurement: $\quad \Gamma\left(D^{0} \rightarrow K^{-} e^{+} v_{\theta}\right)=(9.1 \pm 0.7 \pm$ 1.7) $10^{10} \mathrm{~s}^{-1}$. The two should be equal by isospin symmetry. From the weighted average of the two results, $\Gamma(D \rightarrow K e v)=(7.0 \pm 1.2) \cdot 10^{10} \mathrm{~s}^{-1}$, and using the presently accepted vatue for $\left|V_{c s}\right|$ of $.975( \pm .001)^{15}$, they determine the form factor intercept $\left|f_{+}(0)\right|$ to be $0.69 \pm 0.06$, which is in good agreement with theoretical predictions. The hope is that this knowledge can be turned around later to extract $\left|V_{u b}\right|$. They do point out, however, that the axial form factors as derived from $\Gamma\left(D^{+} \rightarrow K^{\circ} e v\right)(60 \pm$ 15 percent of the scalar rate) do not agree well with theoretical predictions 28 . There is also the question of missing channels as the sum of the scalar and vector decay rates accounts for only $(73 \pm 16) \%$ of the total rate as given by Mark III.

Finally, in a separate publication 29 the E691 collaboration reports on $K 4 \pi$ decays of the $D$ of which the principal results are listed in Table 4. The relatively large value of the second mode is surprising as experiment observes no contribution 
from $\overline{\mathrm{K}}^{* 0} \omega$ decays whereas $\overline{\mathrm{K}}^{* 0} \rho^{0} \pi^{+}$represents a fair fraction of $\mathrm{K}^{-} \pi^{+} \pi^{+} \pi^{+} \pi^{-}$.

\begin{tabular}{|c|c|l|c|}
\cline { 2 - 4 } \multicolumn{1}{c|}{} & \multicolumn{1}{c|}{$A$} & \multicolumn{1}{c|}{$B$} & A B \\
\hline$D^{0}$ & $\bar{K}^{0} \pi^{+} \pi^{+} \pi^{-} \pi^{-}$ & $\bar{K}^{0} \pi^{+} \pi^{-}$ & $0.18 \pm 0.07 \pm 0.04$ \\
\hline$D^{0}$ & $K^{*} \pi^{+} \pi^{+} \pi^{-} \pi^{0}$ & $K^{-} \pi^{+} \pi^{+} \pi^{-}$ & $0.57 \pm 0.06 \pm 0.05$ \\
\hline$D^{+}$ & $K^{-} \pi^{+} \pi^{+} \pi^{+} \pi^{-}$ & $K^{-} \pi^{+} \pi^{+}$ & $0.09 \pm 0.01 \pm 0.01$ \\
\hline
\end{tabular}

Table 4

\section{FRAGMENTATION}

From the observation of $29 \pm 8 \Lambda_{\mathrm{c}}$ charmedbaryons into $\mathrm{pK}^{-} \pi^{+}$, the NA14/2 collaboration makes several comparisons 30 with predictions from the Lund fragmentation program 31 . As stated previously, NA14/2 is a photoproduction experiment with a mean photon energy of $100 \mathrm{GeV}$. From their data they determine the production ratio $\bar{\Lambda}_{c} / \Lambda_{c}$ to be $0.6 \pm 0.3$. One expects this ratio to approach unity for high photon energies ( $>50 \mathrm{GeV}$ ) and for $\Lambda_{c}$ 's to predominate at lower energies. By filtering the Lund Monte Carlo predictions through their apparatus they estimate that a $\bar{\Lambda}_{\mathrm{c}} / \Lambda_{\mathrm{c}}$ ratio of 0.9 should have been observed. No attempt has been made to associate an a priori uncertainty with the Monte Carlo.

Another indication of the inability of the Lund Monte Carlo to reproduce the yield of $\Lambda_{c}$ 's in photoproduction can be seen from NA14/2's observed ratios for $\Lambda_{c} / D^{0}$ and $\Lambda_{c} / D^{+}$. In both cases their results are a factor of 3-4 higher than the Lund predictions. E691 apparently sees a similar discrepancy (the NA14/2 paper assumes a branching ratio for $\Lambda_{c} \rightarrow p K \pi$ of $5 \%$ to allow for a reasonable overestimate of the measured value). Similarly, ARGUS and CLEO apparently see discrepancy factors of around 2 (as analyzed in the NA14/2 paper). A comparison of these results is shown in fig. 5. The conclusion that the Lund program has some difficulties in describing charmed baryon production for photoproduction and $e^{+} e^{-}$ experiments seems inescapable.

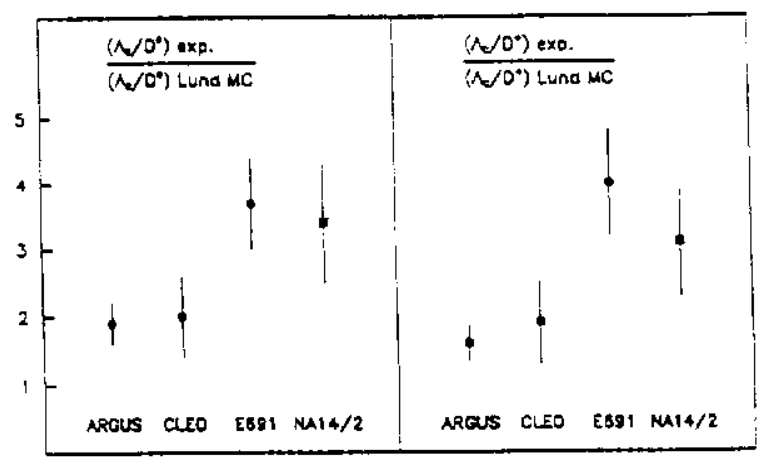

Fig. 5 - Comparison of observed and predicted ratios

\section{RARE STATES}

The NA32 collaboration has published ${ }^{32}$ a first measurement of the $\Xi_{c}^{0}$ lifetime. Their measurement is based on four unambiguous decays into $\mathrm{pK}^{-} \mathrm{R}^{* 0}$ from which a weighted mean mass of $2473.3 \pm 1.9 \pm$ $1.2 \mathrm{MeV} / \mathrm{c}^{2}$ is observed. This mass compares favorably with an eartier observation by CLEO33. The collaboration notes that $\Xi_{c}^{0}$ is apparently heavier than the $\Xi_{c}^{+}$(measured in the same spectrometer) by $6.8 \pm 3.3 \pm 0.5 \mathrm{MeV} / \mathrm{c}^{2}$. After correcting for acceptance, they claim a $\Xi_{c}^{0}$ lifetime of $\left(0.82_{-0.30}^{+0.59}\right) \cdot 10^{\text {. }}$ $13 \mathrm{~s}$. This result is consistent with the theoretical hierarchy of Voloshin and Shifman 34 :

$$
\tau\left(\Omega_{c}^{0}\right)<\tau\left(\Xi_{c}^{0}\right)<\tau\left(\Lambda_{c}^{+}\right) \approx \tau\left(\Xi_{c}^{+}\right)
$$

\section{OPEN BEAUTY}

One experiment, E653, has presented 35 a set of 9 beauty pair events which are derived primarily from a scan of 6320 events $\left(600 \mathrm{GeV} \pi^{-}\right.$beam on an emulsion target). Candidate events were selected at the first level based on the presence of a muon with $\mathrm{P}_{\mathrm{T}}>1.5 \mathrm{GeV} / \mathrm{c}$. Of the eighteen original B's, eleven are neutral and seven charged; one of the neutral B's decays through a $J / \psi$, and three of the neutrals decay through a $D^{*}$. One pair is consistent with mixing in the 
neutral system. It should be emphasized that these events are still under study as well as the systematics of vertex detector and spectrometer.

\section{FUTURE PLANS}

The number of fixed target heavy flavor experiments has been steadily decreasing in recent years. This may be partly due to direct and indirect competition from other High Energy programs. It may also be a concession to the difficulties that confront fixed target experiments as they delve into new areas such as open beauty. Nevertheless, there are fixed target experiments which are presently active and these may be broken down as follows:

Experiments E687 and E791 hope to improve on present charm decay statistics by an order of magnitude. That is, each experiment anticipates a reconstructed charm event sample of order $100 \mathrm{~K}$. Neither experiment precludes the possibility of reconstructing some beauty decays although this is not their main thrust.

The charmonium spectrum continues to be scanned by E760 through the technique described earlier. Special emphasis will be placed on confirming the $\eta_{c}{ }^{\prime}$ and in finding the as yet undiscovered ${ }^{1} P_{1},{ }^{1} D_{2}$, and ${ }^{3} D_{2}$ resonances.

Experiments $E 781$ and WAB9 are similar experiments which intend to use hyperon beams (and pions in the case of E781) to produce and study charmed baryons as well as potential exotic states.

E771, E789, and WA92 will concentrate primarily on hadroproduction of beauty and the weak decay physics of beauty mesons. E771 triggers on either high mass dimuon pairs (to see beauty through the $\mathrm{J} / \Psi$ cascade) and single muons of relatively high transverse momentum. Over the course of the present run and the next fixed target cycle at FNAL (presently scheduled for 1993-94) they hope. assuming a $107 / \mathrm{sec}$ interaction rate can be achieved, to accumulate several hundred completely reconstructed decays and a considerably higher number of partially reconstructed decays. E789 is a very high luminosity spectrometer $\left(10^{8} / \mathrm{sec}\right.$ interaction rate) which is sensitive to two-body decays of charm and beauty. They expect to achieve a sensitivity of $10^{-5}$ in 1991 for $B \rightarrow h^{+} h^{-}$decays and 10.6 in 1993-94. Like E771 and E789, WA92 also uses an extensive silicon microstrip vertex detector for secondary vertex reconstruction. By triggering on vertex information, they hope to accumulate on order of a thousand topological beauty decays.

\section{ACKNOWLEDGEMENTS}

Grateful acknowledgement is made to the U.S. Department of Energy, whose support was invaluable in allowing me to participate the in the Third Topical Conference on Heavy Flavours. I would also like to thank Jeff Appel, Chuck Brown, Joel Butler, Loretta Dauwe, Ron Lipton, Lee Lueking, Ron Ray, Jeff Spalding, Noel Stanton, and Jim Wiss for invaluable discussions on the state of the FNAL experiments.

\section{REFERENCES}

1. T. Matsui and H. Satz, Phys. Lett. 178B (1986) 416.

2. 25th International Conference on High Energy Physics, Singapore, August 1990.

3. L. Rossi, Physics in Collision Conference Proceedings (1989) 43.

4. E672 Collab. S. Kartik et al., Physical Review 410 (1990) 1 .

5. S. Brodsky and A. Mueller, SLAC Report No. NSF-ITP-88.22 (1988) unpublished.

6. E772 Collab., D. M. Alde et al., Phys. Rev. Lett. 64 (1990) 2479.

7. E772 Collab., D. M. Alde et al., Phys. Rev. Lett. 66 (1991) 133.

8. E772 Collab., D. M. Alde et al., Phys. Rev. Lett. 66 (1991) 2285.

9. J. Ashman et al., Phys. Lett. 202B (1988) 603.

10. S. J. Brodsky and P. Hoyer, Phys. Rev. Lett. 63 (1989) 1566. 
11. E687 Collab., P.L. Frabetti et al., Phys. Lett. 251B (1990) 639.

12. E687 Collab., P.L. Frabetti et al., Phys. Lett. 263B (1991) 584.

13. NA14/2 Collab., M. P. Alvarez et al., Z. Phys. $47 \mathrm{C}(1990) 539$.

14. NA32 Collab., S. Bariag et al., Z. Phys. 46C (1990) 563.

15. Particle Data Group, M. Aguilar-Benitez et al., Phys. Lett. 239B (1990) 1

16. E760 Collab., T. A. Armstrong et al., submitted to Nuc. Phys.

17. NA32 Collab., S. Barlag et al., Z. Phys. 49C (1991) 555.

18. E653 Collab., K. Kodama et al., Phys. Lett. 263B (1991) 573.

19. NA32 Collab., S. Barlag et al., Phys. Lett. 257B (1991) 519.

20. B.L. Combridge, Nucl. Phys. 151B (1979) 427;

C.E. Carlson and R. Suaya, Phys. Lett. 81B (1979) 329;

R. Winder and C. Michael, Nucl. Phys. 173B (1980) 59;

V. Barger et al. Phys. Rev. 25D (1982) 112;

R. Odorico, Nuc. Phys. 209B (1982) 77.

21. R. M. Godbole and D.P. Roy, Phys. Rev. Lett. 48 (1982) 1711;

D.P. Roy and B.R. Desai, Z. Phys. 22C (1984) 149.

22. E653 Collab., K. Kodama et al., Phys. Lett. 2638 (1991) 579

23. E. Eichten et al., Rev. Mod. Phys. 58 (1986) 1065.

R.K. Ellis, Fermilab preprint FERMILAB-Conf89/168-T (1989).

24. E691 Collab., J. C. Anjos et al., Phys. Rev. 43D (1991) 635

25. E691 Collab., J. C. Anjos et al., submitted to Phys. Rev. Lett.

26. A. Buras, J.-M. Gérard, R. Rückel, Nucl. Phys. 2688 (1986) 16: M. Bauer, B. Stech, M. Wirbel, Z. Phys. 34C (1987) 103.

27. E691 Collab., J.C. Anjos et al., submitted to Phys. Rev. Lett.
28. E691 Collab., J. C. Anjos et al., Phys. Rev. Lett. 65 (1990) 2630 .

29. E691 Collab., J. C. Anjos et a!., Phys. Rev. 42D (1990) 2414.

30. NA14/2 Collab., M. P. Alvarez et al., Phys. Lett. 246B, (1990) 256.

31. T. Sjöstrand and M. Bengtsson, Comput. Phys. Commun. 43 (1987) 367.

32. NA32 Collab., S. Abrtag et al., Phys. Lett. 236B (1990) 495

33. CLEO Collab., P. Avery et al., Phys. Rev. Lett. 62 (1989) 863.

34. M. B. Voloshin and M. A. Shifman, Zh. Eksp. Teor. Fiz. 91 (1986) 1180.

35. D. Potter, Intersections Conf., Tucson (1991). 